\title{
Truncations in the Method of Intermediate Problems for Lower Bounds to Eigenvalues
}

\author{
Norman W. Bazley and David W. Fox ${ }^{1}$
}

(February 10, 1961)

\begin{abstract}
Two new procedures are developed for determining lower bounds to the eigenvalues of linear operators. The methods are based on the theory of semibounded self-adjoint operators in separable Hilbert space. Computation of the lower bounds is reduced to the solution of matrix problems. The procedures have immediate application in the estimation of eigenvalues and eigenvectors of differential operators appearing in quantum mechanics.
\end{abstract}

\section{Introduction}

In this paper we give two new procedures for determining lower bounds to eigenvalues of semibounded, self-adjoint operators. These procedures, while phrased in the language of Hilbert space, result in useful computational methods for the estimation of eigenvalues and eigenvectors of differential operators occurring in classical and quantum mechanics. Our procedures are based on the method of intermediate problems.

Intermediate problems, as introduced by A. Weinstein [7], ${ }^{2}$ give lower bounds by changing the boundary conditions of differential operators. By relaxing the boundary conditions he obtains a solvable base problem that gives rough lower bounds to the eigenvalues. He then links the base problem to the given problem by a sequence of intermediate problems which can be solved in terms of the base problem and which improve the lower bounds.

Years ago $H$. Weyl [8] pointed out that rough lower bounds can also be obtained by changing the operator. This fact was used by N. Aronszajn [1] to construct intermediate problems for those cases in which a base problem can be obtained by neglecting a positive term in the operator. In the same paper Aronszajn systematized the method of intermediate problems for completely continuous operators in Hilbert space. As Aronszajn points out, the solution of the intermediate problems in his formulation requires the determination of the poles and zeros of a meromorphic function usually given as an infinite sum.

Recently H. F. Weinberger showed that it is possible to overcome the principal difficulties that arise in the intermediate problems of Weinsteint, Weinberger's result, which is a special case of his more general theory [6], gives lower bounds to the eigenvalues by the computation of the zeros of a polynomial.

In this paper we first sketch Aronszajn's method of intermediate problems for changes in the operator. Our presentation allows for the presence of continu-

1 Applied Physics Laboratory, The Johns Hopkins University, Silver Spring, Md.

2 Figures in brackets indicate the literature references at the end of this paper. ous spectra and is in a form suitable for application to many quantum mechanical problems. In the second section we show how it is possible to modify the method so that lower bounds can be obtained by elementary calculations. These results give formulas parallel to some of those of Weinberger [6]. A feature of our procedure is that it can be used to calculate lower bounds to the energy levels of most atomic systems. The last section extends another method of one of the authors [2].

Part of the work presented here was started while the authors were in the Institute for Fluid Dynamics and Applied Mathematics. University of Maryland, and was summarized in [3].

\section{Method of Intermediate Problems}

Let us suppose that $\mathscr{H}$ is a separable Hilbert space with the inner product $(u, v)$ and that $A$ is a selfadjoint operator with domain $\mathscr{D}_{A}$ in $\mathscr{R}$. We will assume $A$ is bounded below and that the first part of its spectrum is discrete. The eigenvalues and eigenvectors in this part of the spectrum will be denoted by $\lambda_{\nu}$ and $u_{\nu}(\nu=1,2, \ldots)$ respectively; the first limit point of the spectrum will be called $\lambda_{\infty}$. Thus we have

$$
A u_{\nu}-\lambda_{\nu} u_{\nu}=0, \quad(\nu=1,2, \ldots)
$$

and

$$
\lambda_{1} \leq \lambda_{2} \leq \ldots \leq \lambda_{\infty} .
$$

Suppose further that $A$ can be written as the sum of two operators,

$$
A=A^{0}+A^{\prime},
$$

where $A^{0}$ is a self-adjoint operator with a known spectral resolution $E_{\lambda}^{0}$ and $A^{\prime}$ is symmetric and positive. The domains of $A^{0}$ and $A^{\prime}$ are denoted by

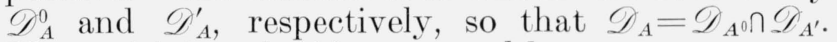
The positivity of $A^{\prime}$ is expressed by

$$
\left(A^{\prime} u, u\right) \geq 0
$$


for every $u$ in the domain of $A^{\prime}$. Finally we assume that the first part of the spectrum of $A^{0}$ is discrete, and, listed in increasing order, the eigenvalues are

$$
\lambda_{1}^{0} \leq \lambda_{2}^{0} \leq \ldots
$$

The corresponding eigenfunctions are $u_{1}^{0}, u_{2}^{0}, \ldots$. .

Since $A^{0} \leq A$, it follows ${ }^{3}[4$, p. 214] that

$$
\lambda_{\nu}^{0} \leq \lambda_{\nu}, \quad(\nu=1,2, \ldots)
$$

and

$$
\lambda_{\infty}^{0} \leq \lambda_{\infty} .
$$

These inequalities go back to H. Weyl [8]. Thus the known eigenvalues of $A^{0}$ give rough lower bounds for the initial eigenvalues of $A$. For this reason the operator $A^{0}$ is called a base operator and the eigenvalue problem for $A^{0}$ is called a base problem.

The procedure of intermediate problems links the base operator $A^{0}$ to the given operator $A$ by a sequence $\left\{A^{k}\right\}$ of intermediate operators. These operators satisfy the inequalities

$$
A^{0} \leq A^{k} \leq A^{k+1} \leq A, \quad(k=1,2, \ldots) ;
$$

and consequently their eigenvalues give intermediate lower bounds to those of $A$.

In order to define these intermediate operators, we introduce an auxiliary Hilbert space $\mathscr{H}^{\prime}$. $\mathscr{H}^{\prime}$ is just the completion of $\mathscr{D}_{A^{\prime}}$ in the norm generated by the inner product $[u, v]$ defined by

$$
[u, v]=\left(A^{\prime} u, v\right) \text {. }
$$

For simplicity we will assume that $A^{\prime}$ is positive definite; the indefinite case requires only a slight extension of the theory. Let $\left\{p_{i}\right\}$ be a linearly independent set of vectors in $\mathscr{D}_{A^{\prime}}$; note that since $A^{\prime}$ is positive definite the sequence $\left\{A^{\prime} p_{i}\right\}$ is also linearly independent. Define $P^{k}$ to be the projection in $\mathscr{H}^{\prime}$ on the span $\mathscr{S}^{k}$ of the inrst $k$ elements of $\left\{p_{i}\right\}$. For an arbitrary vector $v$ in $\mathscr{H}^{\prime}, P^{k} v$ is given by

$$
P^{k} v=\sum_{i, j=1}^{k}\left[v, p_{i}\right] b_{i j} p_{j},
$$

where the constants $b_{i j}$ are the elements of the matrix inverse to that with the elements $\left(A^{\prime} p_{i}, p_{j}\right)$. Since the subspaces $\mathscr{S}^{k}$ are increasing with $k$; i.e.,

$$
\mathscr{P}^{k} \subset \mathscr{P}^{k+1} \subset \mathscr{H}^{\prime}, \quad(k=1,2, \ldots),
$$

the corresponding projections satisfy the inequalities,

$$
0 \leq P^{k} \leq P^{k+1} \leq I
$$

where $I$ is the identity operator in $\mathscr{P}^{\prime}$. We now form the operator $A^{\prime} P^{k}$, which is well defined on

\footnotetext{
${ }^{3} A^{0} \leq A$ means $\mathscr{D}_{A^{0}} \supset \mathscr{D}_{A}$ and $\left(A^{0} u, u\right) \leq(A u, u)$ for every $u$ in $\mathscr{D}_{A}$.
}

$\mathscr{D}_{A}^{\prime}$. For an arbitrary vector $w$ in $\mathscr{D}_{A^{\prime}}, A^{\prime} P^{k} w$ is given

by $A^{\prime} P^{k} w=\sum_{i, j=1}^{k}\left(w, A^{\prime} p_{i}\right) b_{i j} A^{\prime} p_{j}, \quad(k=1,2, \ldots)$.

In the form given on the right in eq (12) $A^{\prime} P^{k}$ has an obvious extension to all of $\mathscr{H}$, and there it is a symmetric completely continuous operator. Henceforward we will regard $A^{\prime} P^{k}$ as thus extended.

It follows from eq (11) that in $\mathscr{H}$ the operators $A^{\prime} P^{k}$ satisfy the inequalities,

$$
0 \leq A^{\prime} P^{k} \leq A^{\prime} P^{k+1} \leq A^{\prime}, \quad(k=1,2, \ldots) .
$$

The intermediate operators $A^{k}$ are now defined by

$$
A^{k}=A^{0}+A^{\prime} P^{k}, \quad(k=1,2, \ldots) ;
$$

$k$ is called the order of the intermediate operator. Since the operators $A^{\prime} P^{k}$ are symmetric and bounded, it follows [5, p. 301] that the operators $A^{k}$ are selfadjoint and have the same domain as $A^{0}$. And moreover, since $A^{\prime} P^{k}$ is for each $k$ a completely continuous operator, each of the operators $A^{k}$ has exactly the same limit points in its spectrum as does $A^{0}[5$, p. 367]. From eq (13) it follows directly that

$$
A^{0} \leq A^{k} \leq A^{k+1} \leq A, \quad(k=1,2, \ldots) .
$$

These inequalities are just those stated in (7), and they show that the operators $A^{k}$ defined by eq (14) are in fact intermediate operators. This means that in the initial discrete part of the spectra the eigenvalues satisfy the inequalities

$$
\lambda_{\nu}^{0} \leq \lambda_{\nu}^{k} \leq \lambda_{\nu}^{k+1} \leq \lambda_{\nu}, \quad(\nu=1,2, \ldots ; \infty),
$$

where the superscripts on the eigenvalues indicate the order of the intermediate operator to which they belong.

We now turn to the problem of determining the eigenvalues and eigenvectors of the intermediate operators $A^{k}$. We will confine our attention to those eigenvalues which lie below the first limit point $\lambda_{\infty}^{0}$ common to all operators $A^{k}$.

The eigenvalue equation for $A^{k}$,

$$
A^{k} u-\lambda u=0,
$$

can be written in a more useful form by using the defining eq (14) for $A^{k}$. This gives

$$
A^{0} u-\lambda u=-A^{\prime} P^{k} u
$$

and by using eq (12) we obtain

$$
A^{0} u-\lambda u=-\sum_{i, j=1}^{k}\left(u, A^{\prime} p_{i}\right) b_{i j} A^{\prime} p_{j}
$$

For convenience we designate the quantities $\sum_{i=1}^{k}$ $\left(u, A^{\prime} p_{i}\right) b_{i j}$ by $\alpha_{j}$, so that the equations to be satis- 
fied become

$$
A^{0} u-\lambda u=-\sum_{j=1}^{k} \alpha_{j} A^{\prime} p_{j}
$$

and

$$
\sum_{j=1}^{k} \alpha_{j}\left(p_{j}, A^{\prime} p_{l}\right)=\left(u, A^{\prime} p_{l}\right), \quad(l=1,2, \ldots, k) .
$$

If $u$ is to be an eigenvector corresponding to a value $\lambda$ which is not in the spectrum of $A^{0}$, then $u$ may be written

$$
u=-\sum_{j=1}^{k} \alpha_{j} R_{\lambda} A^{\prime} p_{j},
$$

where $R_{\gamma}$ is the resolvent operator for $A^{\circ}$.

The eqs (20) become

$$
\sum_{j=1}^{k} \alpha_{j}\left(p_{j}+R_{\lambda} A^{\prime} p_{j}, A^{\prime} p_{l}\right)=0, \quad(l=1,2, \ldots, k) .
$$

All of the $\alpha$ 's cannot vanish if $u$ is to be nontrivial; therefore $\lambda$ must be such that the determinental equation

$$
\left|\left(p_{j}+R_{\lambda} A^{\prime} p_{j}, A^{\prime} p_{l}\right)\right|=0
$$

is satisified.

Equations (22) have solutions for each value $\lambda$ that is not in the spectrum of $A^{\circ}$ and that satisines eq (23). The number of solutions corresponding to each such root is just the nullity of the coefficient matrix and, according to eq (21), these solutions give the eigenvectors of $\mathrm{A}^{k}$ associated with that root. These eigenvectors are clearly linearly independent since $R_{\lambda}$ is one-to-one on $\mathscr{H}$.

For each value $\lambda$ that is an eigenvalue of the operator $\mathbf{A}^{\circ}$, say $\lambda_{\nu}^{0}$, the procedure is somewhat different. Let $\mathbb{K}_{\lambda_{\nu}^{0}}$ be the characteristic subspace of $A^{\circ}$ at the eigenvalue $\lambda_{\nu}^{0}$. Suppose that it is spanned by the eigenvectors $\left\{v_{1}, v_{2}, \ldots, v_{l}\right\}$. It is clear that if $u$ is an eigenvector of $A^{k}$ corresponding to $\lambda_{v}^{0}$, then in addition to the eq (19) and (20) the further restrictions,

$\sum_{j=1}^{k} \alpha_{j}\left(A^{\prime} p_{j}, v_{i-k}\right)=0, \quad(i=k+1, k+2, \ldots, k+l)$

must be satisfied. If we assume that eq (24) are satisfied, $u$ is given by

$$
u=-\sum_{j=1}^{k} \alpha_{j} R_{\lambda_{\nu}^{\prime}}^{\prime} A^{\prime} p_{j}-\sum_{j=k+1}^{k+l} \alpha_{j} v_{j-k},
$$

where by $R_{\lambda_{\nu}^{0}}^{\prime}$ we mean the restriction of the resolvent operator for $\mathrm{A}^{0}$ to the part of our Hilbert space orthogonal to $\mathscr{C}_{\lambda_{v}}$. The eqs (20) become

$$
\begin{array}{r}
\sum_{j=1}^{k} \alpha_{j}\left(p_{j}+R_{\lambda_{\nu}}^{\prime} A^{\prime} p_{j}, A^{\prime} p_{i}\right)+\sum_{j=k+1}^{k+l} \alpha_{j}\left(v_{j-k}, A^{\prime} p_{i}\right)=0, \\
(i=1,2, \ldots, k) .
\end{array}
$$

These, together with eq (24), determine the constants $\alpha_{i}$. The necessary and sufficient condition for the existence of nontrivial solutions is the vanishing of the determinant of the coefficients; i.e.,

$$
\operatorname{det}\left(\begin{array}{c:c}
\left(p_{j}+R_{\lambda_{\nu}^{0}}^{\prime} A^{\prime} p_{j}, A^{\prime} p_{i}\right) & \left(v_{j-k}, A^{\prime} p_{i}\right) \\
\hdashline\left(A^{\prime} p_{j}, v_{i-k}\right) & 0
\end{array}\right)=0 .
$$

Let us assume that (27) holds; that is, that $\lambda_{\nu}^{0}$ is an eigenvalue of $A^{k}$ as well as of $A^{0}$. The eigenvectors of $A^{k}$ corresponding to $\lambda_{\nu}^{0}$ are then determined in the following way: The eqs (24) and (26) have linearly independent solutions $\left\{\alpha_{1}^{\sigma}, \alpha_{2}^{\sigma}, \ldots, \alpha_{k+l}^{\sigma}\right\}, \quad(\sigma=$ $1,2, \ldots, n$ ), where $n$ is the nullity of the coefficient matrix displayed in (27). By (25) these give rise to $n$ vectors $u^{\sigma}$ that are eigenvectors of $A^{k}$ for the value $\lambda_{\nu}^{0}$. These are also linearly independent, for if $C_{\sigma}$ are constants such at $\Sigma_{\sigma=1}^{n} C_{\sigma} u^{\sigma}=0$, then by the expression (25) for $u^{\sigma}$ we must have

$$
R_{\lambda_{\nu}^{\prime}}^{\prime}\left(\sum_{\sigma=1}^{n} C_{\sigma} \sum_{j=1}^{k} \alpha_{j}^{\sigma} A^{\prime} p_{j}\right)+\sum_{\sigma=1}^{n} C_{\sigma} \sum_{j=k+1}^{k+l} \alpha_{j}^{\sigma} v_{j-k}=0 .
$$

But since $R_{\lambda_{\nu}^{0}}^{\prime}$ is a one-to-one operator on the orthogonal complement of $\mathscr{C}_{\lambda_{v}}$, eq (28) implies the separate statements,

$$
\sum_{j=1}^{k}\left(\sum_{\sigma=1}^{n} C_{\sigma} \alpha_{j}^{\sigma}\right) A^{\prime} p_{j}=0, \quad \sum_{j=k+1}^{k+l}\left(\sum_{\sigma=1}^{n} C_{\sigma} \alpha_{j}^{\sigma}\right) v_{j-k}=0 .
$$

The linear independence of the vectors $A^{\prime} p_{i}$ and of the vectors $v_{j}$ imply that

$$
\sum_{\sigma=1}^{n} C_{\sigma} \alpha_{j}^{\sigma}=0, \quad(j=1,2, \cdots, k+l) .
$$

But since the vectors $\left\{\alpha_{1}^{\sigma}, \alpha_{2}^{\sigma}, \ldots, \alpha_{k+l}^{\sigma}\right\}$ are linearly independent, each of the constants $C_{\sigma}$ must vanish, and thus the vectors $u^{\sigma}$ are linearly independent.

As we observed previously, the part of the spectrum of $A^{k}$ that lies below $\lambda_{\infty}^{0}$ is discrete. The eigenvalues there are completely determined from eqs (23) and (27), and the eigenvectors are found from corresponding solutions of the linear equations. These eigenvalues give intermediate lower bounds to those in the first part of the spectrum of $A$.

For the sake of completeness and later use, we sketch a proof of convergence of the eigenvalues of the intermediate problems. The conditions that we use are those stated by Aronszajn [1]. We assume that $A^{0}$ and $A$ have completely continuous inverses, that $A^{\prime}$ is bounded relative to $A^{0}$, and that the set $\left\{p_{i}\right\}$ is complete in $\mathscr{H}^{\prime}$. For convenience we will assume that $A^{0}$ is positive deinite; the modifications for $A^{0}$ bounded below are straightforward.

The purpose of the proof is to show that the inverse operators converge uniformly to $A^{-1}$. From this the convergence of the eigenvalues follows immediately [5, p. 372]. 
Since the inverse operators $\left(A^{k}\right)^{-1}$ form a decreasing sequence of symmetric operators bounded below by $A^{-1}$, the sequence converges strongly to some symmetric operator [5, p. 263]. The strong convergence to $A^{-1}$ is guaranteed by the inequalities that hold for any $t$ in $\mathscr{H}$,

$$
\begin{aligned}
\left\|\left[\left(A^{k}\right)^{-1}-A^{-1}\right] t\right\|^{4} & \\
& \leq\left\|\left(A^{\circ}\right)^{-1}-A^{-1}\right\|^{3}\|t\|^{2}\left(\left[\left(A^{k}\right)^{-1}-A^{-1}\right] t, t\right),
\end{aligned}
$$

and

$$
\begin{array}{r}
\left(\left[\left(A^{k}\right)^{-1}-A^{-1}\right] t, t\right)^{2} \leq\left[\left(I-P^{k}\right) A^{-1} t,\left(I-P^{k}\right) A^{-1} t\right] \\
\cdot\left(A^{\prime}\left(A^{k}\right)^{-1} t,\left(A^{k}\right)^{-1} t\right) .
\end{array}
$$

The first of these inequalities follows by using the Schwarz inequality in the form generated by $\left(A^{k}\right)^{-1}-$ $A^{-1}$ and then using the fact that $\left(A^{k}\right)^{-1} \leq\left(A^{0}\right)^{-1}$. The second is obtained by writing $\left(A^{k}\right)^{-1}-A^{-1}=$ $\left(A^{k}\right)^{-1} A^{\prime}\left(I-P^{k}\right) A^{-1}$, and then using the Schwarz inequality in the form generated by $A^{\prime}$. The first factor on the right in (31) vanishes with increasing $k$ since the set $\left\{p_{i}\right\}$ is complete in $\mathscr{H}^{\prime}$. The second remains bounded since $A^{\prime}$ is bounded relative to $A^{0}$. The strong convergence of $\left(A^{k}\right)^{-1}$ to $A^{-1}$ is thus insured. However, since each operator $\left(A^{k}\right)^{-1}$ as well as $A^{-1}$ is completely continuous and symmetric, the monotonic strong convergence implies uniform convergency by an easily proved analog of Dini's theorem.

REMARK: We observe that if the span of the vectors $\left\{p_{i}\right\}_{i=1}^{k}$ contains some eigenvectors $\left\{u_{\nu_{1}}, u_{\nu_{2}}\right.$ $\left.\ldots, u_{\nu_{j}}\right\}$ of $A,(j \leq k)$, then $A^{k}=A$ on the span of these $u$ 's so that $\lambda_{\nu_{1}}, \lambda_{\nu_{2}}, \ldots, \lambda_{\nu_{j}}$ are eigenvalues of $A^{k}$ as well as $A$, and they have the corresponding eigenvectors $u_{\nu_{1}}, u_{\nu_{2}}, \ldots, u_{\nu_{i}}$, respectively. Investigations based on this property lead to statements of the following type:

Theorem: Let $A$ be an operator with a purely point spectrum and let $\lambda_{\nu}$ be an eigenvalue of $A$ that is less than $\lambda_{\infty}^{0}$, then there exists an intermediate problem (of sufficiently high order) which has $\lambda_{\mu}^{k}=\lambda_{\mu}$ and $u_{\mu}^{k}=u_{\mu}$ for $\mu=1,2, \ldots, \nu$.

\section{Truncation of the Base Operator}

The principal difficulties in the computation of approximating eigenvalues and eigenvectors by the method just described arise from the fact that the resolvent operator $R_{\lambda}$ for the base problem rarely is known in closed form. In most cases it can be expressed only by use of infinite sums or integrals; i.e., in our cases by

$$
R_{\lambda} w=\sum_{\nu=1} \frac{\left(w, u_{\nu}^{0}\right) u_{\nu}^{0}}{\lambda_{\nu}^{0}-\lambda}+\int_{\lambda_{\infty}^{0}-0}^{\infty} \frac{d E_{\mu}^{0} w}{\mu-\lambda} .
$$

We overcome this difficulty by changing the base problem so that it has a simple resolvent and still provides lower bounds by the method of intermediate problems. These bounds are computed from equations identical with those given earlier ex- cept that the resolvent is expressed by a finite sum with a remainder in closed form.

Let us assume that $A^{0}$ has infinitely many eigenvalues less than $\lambda_{\infty}$ (the modifications for the cases in which there are only a finite number will be immediate). Define the truncated operators $A^{i, 0}$ by the equations

$$
A^{i, 0}=A^{0} E_{\lambda_{i}^{0}}^{0}+\lambda_{i+1}^{0}\left(I-E_{\lambda_{i}^{0}}^{0}\right), \quad(i=1,2, \ldots),
$$

and denote the corresponding resolutions of the identity by $E_{\lambda}^{i, 0}$. It is clear that each operator $A^{i, 0}$ is bounded and symmetric, has the eigenvalues and eigenvectors $\lambda_{\nu}^{o}, u_{\nu}^{o}$ for $\nu=1,2, \ldots, i$, and also has $\lambda_{i+1}^{o}$ as an eigenvalue of infinite multiplicity. The resolutions of the identity may be written down immediately. They are

$$
E_{\lambda}^{i, 0}=\left\{\begin{array}{ll}
E_{\lambda}^{0}, & \lambda<\lambda_{i+1}^{0} \\
I, & \lambda_{i+1}^{0} \leq \lambda
\end{array}\right\}, \quad(i=1,2, \ldots) .
$$

An operator $A^{i, 0}$ is called a truncation of $A^{0}$ of order $i$. These operators satisfy the fundamental inequalities

$$
A^{i, 0} \leq A^{i+1,0} \leq A^{0}, \quad(i=1,2, \ldots),
$$

since from the spectral theorem it follows that

$$
\left(\left[A^{i+1,0}-A^{i, 0}\right] u, u\right)=\left(\lambda_{i+2}^{0}-\lambda_{i+1}^{0}\right) \int_{\lambda_{i+2}^{0}-0}^{\infty} d\left(E_{\lambda} u, u\right) \geq 0 .
$$

for each $u$ in $\mathscr{H}$, and that

$$
\left(\left[A^{0}-A^{i, 0}\right] u, u\right)=\int_{\lambda_{i+1}^{0}-0}^{\infty}\left(\lambda-\lambda_{i+1}^{0}\right) d\left(E_{\lambda}^{0} u, u\right) \geq 0
$$

for each $u$ in $\mathscr{D}_{A^{0}}$.

The new intermediate operators $A^{i, k}$ based on $A^{i, 0}$ are defined by

$$
A^{i, k}=A^{i, 0}+A^{\prime} P^{k}, \quad(i, k=1,2, \ldots) .
$$

These are bounded, symmetric, and are monotonically increasing in $i$ and $k$; i.e.,

$$
A^{i, k} \leq A^{i+1, k} \leq A^{k} \leq A, \quad(i, k=1,2, \ldots),
$$

and

$$
A^{i, k} \leq A^{i, k+1} \leq A^{i, 0}+A^{\prime} \leq A, \quad(i, k=1,2, \ldots .) .
$$

The montonicity in the first index follows from (34) and in the second index from (13). The inequalities (37) and (38) imply the parallel inequalities for the corresponding eigenvalues,

$$
\left.\begin{array}{l}
\lambda_{\nu}^{i, k} \leq \lambda_{\nu}^{i+1, k} \leq \lambda_{\nu}^{k} \leq \lambda_{\nu} \\
\lambda_{\nu}^{i, k} \leq \lambda_{\nu}^{i, k+1} \leq \lambda_{\nu}
\end{array}\right\}, \quad(\nu=1,2, \ldots),
$$


where by $\lambda_{v}^{i, k}$ we mean the $\nu$ th eigenvalue of $A^{i, k}$.

Since $A^{i, k}$ and $A^{i, 0}$ differ by a completely continuous operator, the limit point $\lambda_{i+1}^{0}$ persists and is the only limit point of the spectrum of the intermediate operator $A^{i, k}$ as the order $k$ of the operator is increased [5, p. 367]. Further, the inequalities (39) show that for any $k$ there are at most $i$ eigenvalues of $A^{i, k}$ less than $\lambda_{i+1}^{0}$.

The spectrum of each operator $A^{i, k}$ can be determined easily by the usual procedure for intermediate problems. The eigenvalue equation for $A^{i, k}$ is stated by

$$
A^{i, k} u-\lambda u=0,
$$

or equivalently (in the notation of the preceding section)

$$
\begin{gathered}
A^{i, 0} u-\lambda \iota=-\sum_{j=1}^{k} \alpha_{j} A^{\prime} p_{j}, \\
\sum_{i=1}^{k} \alpha_{j}\left(p_{j}, A^{\prime} p_{l}\right)=\left(u, A^{\prime} p_{l}\right), \quad(l=1,2, \ldots, k) .
\end{gathered}
$$

If $u$ is an eigenvector corresponding to a value $\lambda$ that is not in the spectrum of $A^{i, 0}$, then $u$ may be written

$$
u=-\sum_{j=1}^{k} \alpha_{j} R_{\lambda}^{i} A^{\prime} p_{j}
$$

where $R_{\lambda}^{i}$ is the resolvent operator of $A^{i, 0}$. We note here that for any vector $v$ in $\mathscr{H}, R_{\lambda}{ }^{i} v$ is given by the closed expression,

$$
R_{\lambda}^{i} v=\sum_{\nu=1}^{\imath} \frac{\left(v, u_{\nu}^{0}\right) u_{\nu}^{0}}{\lambda_{\nu}^{0}-\lambda}+\frac{1}{\lambda_{i+1}^{0}-\lambda}\left[v-\sum_{\nu=1}^{i}\left(v, u_{\nu}^{0}\right) u_{\nu}^{0}\right] .
$$

The eqs (22) become

$$
\sum_{i=1}^{k} \alpha_{j}\left(p_{j}+R_{\lambda}^{i} A^{\prime} p_{j}, A^{\prime} p_{l}\right)=0, \quad(l=1,2, \cdots, k) .
$$

As a consequence, the necessary and sufficient condition for such a $\lambda$ to be an eigenvalue is that it be a root of the equation

$$
\left|\left(p_{j}+R_{\lambda}^{i} A^{\prime} p_{j}, A^{\prime} p_{l}\right)\right|=0 .
$$

The multiplicity of each such eigenvalue is the nullity of the coefficient matrix of eq (42). The eigenvectors of $A^{i, k}$ are determined from the solutions of eq (42) by eq (40).

Now suppose that $\lambda_{\nu}^{0}$ is an eigenvalue of $A^{i, 0}$ that is less than $\lambda_{i+1}^{0}$ and that the characteristic subspace $\mathscr{U}_{\lambda_{\nu}^{0}}^{0}$ of $A^{i, 0}$ corresponding to $\lambda_{\nu}^{0}$ is spanned by $\left\{v_{1}, v_{2}, \ldots, v_{l}\right\}$. If $u$ is to be an eigenvector of
$A^{i, k}$ corresponding to $\lambda_{\nu}^{0}$, then $u$ is given by the equation

$$
u=-\sum_{j=1}^{k} \alpha_{j} R_{\lambda_{\nu}^{0}}^{i \prime} A^{\prime} p_{j}-\sum_{j=k+1}^{k+l} \alpha_{j} v_{j-k},
$$

where the constants $\alpha_{j}$ must satisfy the equations

$$
\begin{aligned}
& \sum_{j=1}^{k} \alpha_{j}\left(p_{j}+R_{\lambda_{\nu}^{0}}^{\lambda^{\prime}} A^{\prime} p_{j}, A^{\prime} p_{r}\right) \\
& +\sum_{j=k+1}^{k+l} \alpha_{j}\left(v_{j-k}, A^{\prime} p_{r}\right)=0, \quad(r=1,2, \ldots, k)
\end{aligned}
$$

and

$\sum_{j=1}^{k} \alpha_{j}\left(A^{\prime} p_{j}, v_{r-k}\right)=0, \quad(r=k+1, k+2, \ldots, k+l)$.

The linear independence of the eigenvectors arising from linearly independent sets of $\alpha$ 's may be demonstrated as in the preceding section.

The multiplicity of $\lambda_{\nu}^{0}$ as an eigenvalue of $A^{i, k}$ is thus just the nullity of the matrix

$$
\left(\begin{array}{c:c}
\left(p_{j}+R_{\lambda_{\nu}^{0}}^{i^{\prime}} A^{\prime} p_{j}, A^{\prime} p_{r}\right) & \left(v_{j-k}, A^{\prime} p_{r}\right) \\
\hdashline\left(A^{\prime} p_{j}, v_{r-k}\right) & 0
\end{array}\right) .
$$

We have found all of the eigenvalues of $A^{i, k}$ that are not equal to $\lambda_{i+1}^{0}$. The complete continuity of $A^{\prime} P^{k}$ implies that $\lambda_{i+1}^{0}$, the only limit point of the spectrum of $A^{i, 0}$, remains as the only limit point of the spectrum of $A^{i, k}$. Thus the total spectrum of $A^{i, k}$ is determined. The spectral subspace of $A^{i, k}$ associated with $\lambda_{i+1}^{0}$ is just the orthogonal comple. ment of the span of all the eigenvectors already determined for the values of $\lambda$ not equal to $\lambda_{i+1}^{0}$.

Thus, as stated at the beginning of this section, the method of truncation justifies the replacement of the base problem resolvent by a suitable approximation in closed form. The necessary computations can be carried out by routine techniques.

The convergence of the eigenvalues of the truncated intermediate operator $A^{i, k}$ to those of $A^{k}$ can be shown under the assumption that $A^{0}$ and $A$ have completely continuous inverses. We demonstrate the uniform convergence of $\left(A^{i, k}\right)^{-1}$ to $\left(A^{k}\right)^{-1}$ for each fixed $k$. For convenience we will assume that $A^{\circ}$ is positive definite. First we note that the operators $\left(A^{i, 0}\right)^{-1}$ converge in norm to $\left(A^{0}\right)^{-1}$ as $i$ becomes infinite. This follows directly from the spectral theorem, for we have

$$
\left(A^{0}\right)^{-1}=\int^{\infty} \lambda^{-1} d E_{\lambda}^{0},
$$

and

$$
\left(A^{i, 0}\right)^{-1}=\int^{\lambda_{i+1}^{0}-0} \lambda^{-1} d E_{\lambda}^{0}+\frac{1}{\lambda_{i+1}^{0}} \int_{\lambda_{i+1}^{0}-0}^{\infty} d E_{\lambda}^{0},
$$


so that

$$
\left(A^{0}\right)^{-1}-\left(A^{i, 0}\right)^{-1}=\int_{\lambda_{i+1}^{0}-0}^{\infty}\left(\frac{1}{\lambda}-\frac{1}{\lambda_{i+1}^{0}}\right) d E_{\lambda}^{0} .
$$

Consequently we have

$$
\left\|\left(A^{0}\right)^{-1}-\left(A^{i, 0}\right)^{-1}\right\| \leq \frac{1}{\lambda_{i+1}^{0}} .
$$

Since $\lambda_{i+1}^{0}$ becomes infinite with $i$, the norm convergence of $\left(A^{i, 0}\right)^{-1}$ is clear. This implies that $\left(A^{i, k}\right)^{-1}$ converges in norm to $\left(A^{k}\right)^{-1}$. The proof goes as follows. We start from the simple identity

$$
A^{i, k}-A^{k}=A^{i, 0}-A^{0} .
$$

From this it follows immediately that

$$
A^{i, k}\left[\left(A^{k}\right)^{-1}-\left(A^{i, k}\right)^{-1}\right] A^{k}=A^{i, 0}\left[\left(A^{0}\right)^{-1}-\left(A^{i, 0}\right)^{-1}\right] A^{0},
$$

and thus

$$
\begin{aligned}
\left(A^{k}\right)^{-1}-\left(A^{i, k}\right)^{-1}= & \left(A^{i, k}\right)^{-1} A^{i, 0} \\
& {\left[\left(A^{0}\right)^{-1}-\left(A^{i, 0}\right)^{-1}\right] A^{0}\left(A^{k}\right)^{-1} . }
\end{aligned}
$$

By writing $\left(A^{i, k}\right)^{-1} A^{i, 0}$ as $I-\left(A^{i, k}\right)^{-1} A^{\prime} P^{k}$ it is evident that $\left(A^{i, k}\right)^{-1} A^{i, 0}$ is an operator that can be bounded independently of $i$ since $\left\|\left(A^{i, k}\right)^{-1}\right\| \leq \frac{1}{\lambda_{1}^{0}}$. Since $A^{0}\left(A^{k}\right)^{-1}$ is also bounded for each fixed $k$, the convergence in norm of $\left(A^{i, k}\right)^{-1}$ to $\left(A^{k}\right)^{-1}$ follows directly from (48) and (49).

The uniform convergence of $\left(A^{i, k}\right)^{-1}$ to $\left(A^{k}\right)^{-1}$ and that of $\left(A^{k}\right)^{-1}$ to $A^{-1}$ demonstrated in section 2 are sufficient to insure that the eigenvalues of $A^{i, k}$ converge to those of $A$ as $i$ and $k$ become large.

\section{Truncation by Choice of Elements}

In this section we discuss an extension of another procedure [2] for truncating the resolvent $R_{\lambda}$ that appears in section 2. This can be accomplished by choosing the elements $p_{i}$ in suitable ways instead of changing the base problem operator as has been done in previous sections.

We assume that the vectors $\left\{p_{1}, p_{2}, \ldots, p_{k}\right\}$ which determine $A^{k}$ have been chosen so that

$$
A^{\prime} p_{i}=\sum_{\nu=1}^{N} \beta_{i \nu} u_{\nu}^{0}, \quad(i=1,2, \ldots, k) .
$$

That is, it is assumed that each of quantities $A^{\prime} p_{i}(i=1,2, \ldots, k)$ can be expressed in terms of a finite number $N$ of eigenvectors of $A^{0}$. The number $N$ will of course depend on $k$. The quantities $\left(R_{\lambda} A^{\prime} p_{i}, A^{\prime} p_{j}\right)$ which appear in section 2 will have the simple expressions

$$
\left(R_{\lambda} A^{\prime} p_{i}, A^{\prime} p_{j}\right)=\sum_{\nu=1}^{N} \frac{\beta_{i \nu} \bar{\beta}_{\nu j}}{\lambda_{\nu}^{0}-\lambda}
$$

and thus are effectively truncated by the choice of elements (50). The computations for the eigenvalues and eigenvectors may be carried out directly from the equations of section 2 .

In the present case, however, it is simpler to proceed directly. We have already shown that the expression $A^{k} u$ can be written

$$
A^{k} u=A^{0} u+\sum_{i, j=1}^{k}\left(u, A^{\prime} p_{i}\right) b_{i j} A^{\prime} p_{j}
$$

where, as before, the quantities $b_{i j}$ are the elements of the matrix inverse to that having $\left(A^{\prime} p_{i}, p_{j}\right)$ as elements. Using (50) this expression becomes

$$
A^{k} u=A^{0} u+\sum_{i, j=1}^{k} \sum_{\mu, \nu=1}^{N}\left(u, u_{\nu}^{0}\right) \bar{\beta}_{i \nu} b_{i j} \beta_{j \mu} u_{\mu}^{0} .
$$

We can now obtain all of the eigenvalues and eigenvectors of $A^{k}$ by inspection. First consider those vectors in $\mathscr{D}_{A^{0}}$ that are orthogonal to every eigenvector of $A^{0}$. If $u$ is such a vector, then (52) shows that $A^{k} u-\lambda u=A^{0} u-\lambda u$. Clearly no such a vector can be an eigenvector of $A^{k}$. Further, if $u$ is an eigenvector $u_{\sigma}^{0}$ of $A^{0}$ with an eigenvalue $\lambda_{\sigma}^{0}$ and is not used in the sums in (50), then by (52) we have $A^{k} u_{\sigma}^{0}-\lambda_{\sigma}^{0} u_{\sigma}^{0}=A^{0} u_{\sigma}^{0}-\lambda_{\sigma}^{0} u_{\sigma}^{0}=0$. Hence each such vector is an eigenvector of $A^{k}$ with the same eigenvalue as for $A_{\sigma}^{0}$.

It remains to consider those vectors $u$ which are linear combinations of the vectors $u_{\nu}^{0}(\nu=1$, $2, \ldots, N)$ used in the sums in $(50)$. There are exactly $N$ linearly independent vectors of this kind that are eigenvectors of $A^{k}$. These are determined as follows. We write $u$ as

$$
u=\sum_{\nu=1}^{N} \gamma_{\nu} u_{\nu}^{0}
$$

and insert this in the eigenvalue equation $A^{k} u-\lambda u=0$, where $A^{k}$ is given by (52). The linear independence of the vectors $u_{\nu}^{0}$ leads to the equivalent algebraic system,

$$
\begin{aligned}
& \sum_{\nu=1}^{N} \gamma_{\nu}\left(\sum_{i, j=1}^{k} \bar{\beta}_{i \nu} b_{i j} \beta_{j \mu}+\left(\lambda_{\nu}^{0}-\lambda\right) \delta_{i \mu}\right)=0, \\
&(\mu=1,2, \ldots, N) .
\end{aligned}
$$

Consequently the determination of the intermediate eigenvalues and eigenvectors depends only on the solution of a matrix eigenvalue problem.

This procedure can be especially useful when the operator $A^{\prime}$ is a multiplicative operator, as for example in the spheriodal wave equation. ${ }^{4}$ In the prolate case the eigenvalue equation is stated by

$$
A u-\lambda u=0,
$$

4 The numerical applications in this and other cases are presently being carried out by W. Börsch-Supan. 
in which $A$ is the essentially self-adjoint operator given by

$$
A u=-\frac{d}{d x}\left[\left(1-x^{2}\right) \frac{d u}{d x}\right]+\frac{m^{2}}{1-x^{2}} u+c^{2} x^{2} u
$$

on the set of all functions continuous on $[-1,+1]$ that are twice continuously differentiable on $(-1,+1)$ and for which $A u$ belongs to $\mathscr{L}^{2}(-1,+1)$. Here $m$ is a non-negative integer and $c$ is a real constant. A base operator is obtained by omitting the positive term $A^{\prime}=c^{2} x^{2}$. The solutions of the base problem are associated Legendre functions. The choice $p_{i}=u_{i}^{0}=P_{m+i-1}^{m}$ and the recursion relation,

$$
x P_{n}^{m}=\frac{(n-m+1) P_{n+1}^{m}+(n+m) P_{n-1}^{m},}{2 n+1}
$$

allow one to express $A^{\prime} p_{i}$ as a linear combination of $u_{i-2}^{0}, u_{i-1}^{0}, u_{i}^{0}, u_{i+1}^{0}$, and $u_{i+2}^{0}$. Other examples of this sort can be found easily.

\section{References}

[1] N. Aronszajn, Approximation methods for eigenvalues of completely continuous symmetric operators, Proc. Symp. Spectral Theory and Differential Problems (Stillwater, Okla., 1951).

[2] N. W. Bazley, Lower bounds for eigenvalues with application to the helium atom, Proc. Nat. Acad. Sci. 45 (1959).

[3] N. W. Bazley and D. W. Fox, Lower bounds for eigenvalues by explicitly solvable intermediate problems, Am. Math. Soc. Notices 6, 839 (1959).

[4] T. Kato, On the existence of solutions of the helium wave equation, Trans. Am. Math. Soc. 70 (1951).

[5] F. Riesz and B. Sz.-Nagy, Functional Analysis (Frederic Ungar, New York 1955).

[6] H. F. Weinberger, A theory of lower bounds for eigenvalues, Technical Note BN-183 (Institute for Fluid Dynamies and Applied Mathematies, Univ. Maryland, 1959).

[7] A. Weinstein, Études des spectres des équations aux dérivées partielles, Mémorial des Sciences Mathe matiques No. 88 (1937).

[8] H. Weyl, Ueber die Abhängigkeit der Eigenschwingungen einer Membran von deren Begrenzung, J. reine angew. Math. 141 (1912).

(Paper 65B2-48) 